American J. of Engineering and Applied Sciences 1 (4): 324-328, 2008

ISSN 1941-7020

(C) 2008 Science Publications

\title{
Analysis of a Beam Made of Physical Nonlinear Material on Nonlinear Elastic Foundation under a Moving Concentrated Load
}

\author{
E. Mardani \\ Civil Engineering Department of Urmia University, Urmia, Iran
}

\begin{abstract}
A prismatic beam made of a behaviorally nonlinear material was analyzed under a concentrated load moving with a known velocity on a nonlinear elastic foundation with a reaction $\left[\mathrm{q}_{\mathrm{e}}=-\mathrm{K}_{1} \mathrm{~W}\left(1+\mathrm{K}_{2} \mathrm{~W}^{2}\right)\right]$. the vibration equation of motion was derived using Hamilton principle and Euler Lagrange equation. The amplitude of vibration, circular frequency, bending moment, stress and deflection of the beam can be calculated by the presented solution. Considering the response of the beam, in the sense of its resonance, it was found that there is no critical velocity when the behavior of the beam and foundation material is assumed to be physically nonlinear and there are finite values for the deflection, stress and bending moment of the beam when $\eta^{2}=\frac{\theta^{2}}{\omega^{2}}=1$.
\end{abstract}

Key words: Physical nonlinear, moving load, hamilton principle, euler lagrange equation, duffing equation

\section{INTRODUCTION}

Presently there are many structures made from materials which are not subject to the Hook's law. Therefore there is a great tendency to study stress and strain at elements of structures made of physical nonlinear material under various statically and dynamical loads. In linear theory the property of material is not taken into consideration. However, all of relevant parameters are taken into consideration at the theory of nonlinearity. Thus, physical nonlinear theory for small deformations demonstrates an exact calculation method for the analysis of stress, strain and other internal forces at structural elements.

In this connections, Skramtayev, Stolyarov, Tyte, Schlechtwg, Grubbier, Cox and others' have presented formulas concerning the relationships between stress and $\operatorname{strain}^{[3]}$.

Finally the relationship between stress and strain in the case of physical nonlinear is presented by Hans Kaudrer $^{[1]}$.

$\varepsilon_{\mathrm{ij}}=\frac{\mathrm{K}\left(\sigma_{0}\right)}{3 \mathrm{~K}} \sigma_{0}+\frac{1\left(\mathrm{t}_{0}^{2}\right)}{2 \mathrm{G}}\left(\delta_{\mathrm{ij}}-\sigma_{0} \delta_{\mathrm{ij}}\right)$

where, $i, j=1,2,3$

Where $\mathrm{K}$ and $\mathrm{G}$ at small deformation are volume contraction and shear elastic module respectively. $\mathrm{K}(\sigma)$ is average stress function and $1\left(\mathrm{t}_{0}{ }^{2}\right)$ is shear stress function therefore:

$$
\begin{aligned}
& \mathrm{K}\left(\sigma_{0}\right)=1+\mathrm{K}_{1} \sigma_{0}+\mathrm{K}_{2} \sigma_{0}^{2}+\ldots=\sum_{\mathrm{n}=0}^{\infty} \mathrm{K}_{\mathrm{n}} \sigma_{0}^{\mathrm{n}} \\
& 1\left(\mathrm{t}_{0}^{2}\right)=1+1_{2} \mathrm{t}_{0}^{2}+1_{4} \mathrm{t}_{0}^{4}+\ldots=\sum_{\mathrm{n}=0}^{\infty} \mathrm{1}_{2 \mathrm{n}} \mathrm{t}_{0}^{\mathrm{n}}
\end{aligned}
$$

Researches have demonstrated that $\mathrm{K}(\sigma)$ at physical nonlinear material at average relative deformation is close to the straight line $K(\sigma)=1$ and the two first sentences of shear stress function are enough.

$1\left(t_{0}^{2}\right)=1+l_{2} t_{0}^{2}$

Where is the physical nonlinear coefficient. The relationship between stress and strain at beams by simplification of Eq. 1 for two dimensional surfaces is:

$\sigma_{\mathrm{z}}=\mathrm{E}\left(\varepsilon_{\mathrm{z}}-\frac{2}{27} 1_{2} \frac{\mathrm{E}^{3}}{\mathrm{G}^{3}} \varepsilon_{\mathrm{z}}^{3}\right)$

The purpose of this study was the analysis of a beam made of physical nonlinear material under moving load on nonlinear elastic foundation.

\section{MATERIALS AND METHODS}

It is assumed that the moving concentrated load along the simply supported prismatic beam laid on nonlinear elastic foundation shown in Fig. 1. 


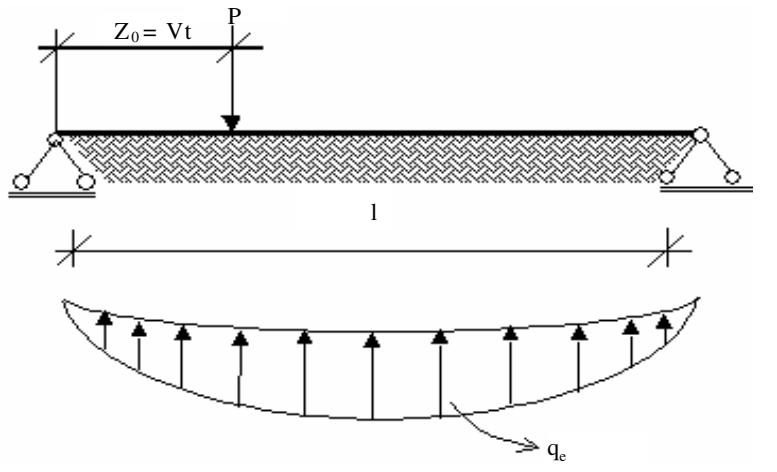

Fig. 1: Schematic view of a prismatic beam under moving load

The load $\mathrm{P}$ moves on the beam with a constant velocity $\mathrm{V}$. The reaction of the nonlinear elastic foundation is:

$$
\mathrm{q}_{\mathrm{e}}-\mathrm{k}_{1} \mathrm{~W}\left(1+\mathrm{k}_{2} \mathrm{~W}^{2}\right)
$$

Where $\mathrm{k}_{1}, \mathrm{k}_{2}$ are coefficients with respect to nonlinear elastic foundation which are determined experimentally, where $\mathrm{W}$ is the beam deflection. follow:

The principle of Hamilton for this beam is as

$$
H=\int_{t_{1}}^{t_{2}}(\Pi-A-E) d t
$$

The potential and kinetic energy of this system can be written as follows.

$$
\begin{aligned}
& \Pi=\int_{0}^{1}\left[\frac{1}{2} \mathrm{EJ}\left[\frac{\partial^{2} \mathrm{~W}}{\partial \mathrm{Z}^{2}}\right]^{2}-\frac{1}{54} \mathrm{l}_{2} \frac{\mathrm{E}^{4}}{\mathrm{G}^{3}} \mathrm{~J}_{1}\left[\frac{\partial^{2} \mathrm{~W}}{\partial \mathrm{Z}^{2}}\right]^{4}-\right. \\
& \left.\frac{1}{2} \mathrm{k}_{1} \mathrm{~W}^{2}-\frac{1}{4} \mathrm{k}_{1} \mathrm{k}_{2} \mathrm{~W}^{4}\right] \mathrm{dz} \\
& \mathrm{E}=\frac{1}{2} \rho \mathrm{F} \int_{0}^{1}\left[\frac{\partial \mathrm{W}}{\partial \mathrm{t}}\right]^{2} \mathrm{dZ}
\end{aligned}
$$
by ${ }^{[4]}$.

The research of the external moving load is given

$$
\begin{aligned}
& \mathrm{q}(\mathrm{z}, \mathrm{t})=\sum_{\mathrm{k}=1}^{\infty} \frac{2 \mathrm{P}}{\mathrm{l}} \sin \frac{\mathrm{k} \pi \mathrm{z}}{\mathrm{l}} \sin \frac{\mathrm{k} \pi \mathrm{z}}{\mathrm{l}} \\
& \mathrm{A}=\int_{0}^{1} \mathrm{q}(\mathrm{z}, \mathrm{t}) \cdot \mathrm{W}(\mathrm{z}, \mathrm{t}) \mathrm{dz}= \\
& \frac{2 \mathrm{P}}{\mathrm{l}} \int_{0}^{1} \sum \sin \frac{\mathrm{k} \pi \mathrm{z}_{\circ}}{\mathrm{l}} \cdot \operatorname{Sin} \frac{\mathrm{k} \pi \mathrm{z}}{1} \mathrm{~W}(\mathrm{z}, \mathrm{t}) \mathrm{dz}
\end{aligned}
$$
have:

By substitution of expressions 7, 8, 10 in 6, we will

$$
\begin{aligned}
& \mathrm{H}=\int_{\mathrm{t}_{1}}^{\mathrm{t}_{2}} \int_{0}^{1}\left[\frac{1}{2} \mathrm{EJ}_{0}\left(\frac{\partial^{2} \mathrm{~W}}{\partial \mathrm{z}^{2}}\right)^{2}-\frac{1}{54} \mathrm{l}_{2} \frac{\mathrm{E}^{4}}{\mathrm{G}^{3}} \mathrm{~J}_{1}\left[\frac{\partial^{2} \mathrm{~W}}{\partial \mathrm{z}^{2}}\right]^{4}\right. \\
& -\frac{1}{2} \mathrm{k}_{1} \mathrm{~W}^{2}-\frac{1}{4} \mathrm{k}_{1} \mathrm{k}_{2} \mathrm{~W}^{4}- \\
& \left.\frac{2 \mathrm{p}}{1} \sum_{\mathrm{k}=1}^{\infty} \sin \frac{\mathrm{k} \pi \mathrm{z}_{0}}{1} \sin \frac{\mathrm{k} \pi \mathrm{z}}{1} \cdot \mathrm{w}(\mathrm{z}, \mathrm{t})-\frac{1}{2} \rho \mathrm{F}\left(\frac{\partial \mathrm{W}}{\partial \mathrm{t}}\right)^{2}\right] \mathrm{dzdt}
\end{aligned}
$$

Substituting, in expression 11 the principle of Hamilton Will be as follows:

$$
\begin{aligned}
& \mathrm{H}=\frac{1}{\pi \omega} \int_{0}^{2 \pi} \int_{0}^{\pi}\left[\frac{\pi^{4}}{21} \mathrm{EJ} \cdot\left(\frac{\partial^{2} \mathrm{~W}}{\partial \xi^{2}}\right)^{2}\right. \\
& \left.-\frac{1}{54} \mathrm{l}_{2} \frac{\pi^{8} \mathrm{E}^{4}}{1^{8} \mathrm{G}^{3}} \mathrm{~J}_{1}\left(\frac{\partial^{2} \mathrm{~W}}{\partial \xi^{2}}\right)^{4}\right]- \\
& \frac{1}{2} \mathrm{k} \mathrm{W}^{2}\left(1+\frac{1}{2} \mathrm{k}_{2} \mathrm{~W}^{2}\right)-\mathrm{W} \frac{2 \mathrm{P}}{1} \sum_{\mathrm{k}=1}^{\infty} \sin \mathrm{k} \xi_{\circ} \cdot \sin \mathrm{k} \xi \\
& -\frac{1}{2} \rho \mathrm{F} \omega \\
& 2
\end{aligned}
$$

The deformation of the beam is found from the following expression:

$\mathrm{W}(\xi, \tau)=\mathrm{p}(\xi) \cdot \mathrm{q}(\tau)$

Where $\mathrm{p}(\xi)$ and $\mathrm{q}(\tau)$ are coordinate and generalized function respectively. Substituting expression 13 in 12 we will have:

$$
\begin{aligned}
& \mathrm{H}=\frac{1}{\pi \omega} \int_{0}^{2 \pi} \int_{0}^{\pi}\left[\frac{1}{2} \mathrm{EJ} \frac{\pi^{4}}{1^{4}}\left[\mathrm{P}^{\prime \prime}(\xi) \mathrm{q}(\mathrm{t})\right]^{2}-\right. \\
& -\frac{1}{54} \mathrm{l}_{2} \frac{\pi^{8} \mathrm{E}^{4}}{\mathrm{l}^{8} \mathrm{G}^{3}} \mathrm{~J}_{1}\left[\mathrm{P}^{\prime \prime}(\xi) \mathrm{q}(\mathrm{t})\right]^{4}- \\
& \mathrm{p}(\xi) \cdot \mathrm{q}(\mathrm{t}) \frac{2 \mathrm{p}}{1} \sum_{\mathrm{k}=1}^{\infty} \operatorname{Sink} \xi_{0} \cdot \operatorname{Sink} \xi- \\
& \left.-\frac{1}{2} \mathrm{pF} \Phi^{2} \mathrm{P}(\xi)^{2} \cdot \mathrm{q}^{\prime}(\mathrm{t})^{2}\right] \mathrm{d} \xi \mathrm{dt}
\end{aligned}
$$

It is assumed that $\mathrm{P}(\xi)$ is known so the Hamilton integral will be as follows: 


$$
\begin{aligned}
H & =\frac{1}{\pi \omega} \int_{0}^{2 \pi}\left[\mathrm{aq}^{2}+\mathrm{bq}^{4}-\mathrm{c \omega} \mathrm{q}^{2} \mathrm{q}^{2}-\mathrm{d}_{1} \mathrm{q}\right] \\
& =\frac{1}{\pi \omega} \int_{0}^{2 \pi} \mathrm{Ld} \tau
\end{aligned}
$$

Where:

$$
\begin{aligned}
& \mathrm{a}=\frac{1}{2} \mathrm{EJ}_{0} \frac{\pi}{1} \int_{0}^{\pi}\left[\mathrm{p}^{\prime \prime}(\xi)\right]^{2} \mathrm{~d} \xi-\frac{1}{2} \mathrm{~K}_{1} \int_{0}^{\pi}[\mathrm{P}(\xi)]^{2} \mathrm{~d} \xi \\
& \mathrm{b}=-\frac{1}{54} \mathrm{l}_{2} \frac{\mathrm{E}^{4}}{\mathrm{G}^{3} \mathrm{~J}_{1}} \frac{\pi^{8}}{1^{8}} \int_{0}^{\pi}\left[\mathrm{p}^{\prime \prime}(\xi)\right]^{4} \\
& \mathrm{~d} \xi-\frac{1}{4} \mathrm{~K}_{1} \mathrm{~K}_{2} \int_{0}^{\pi}[\mathrm{p}(\xi)]^{4} \mathrm{~d} \xi \\
& \mathrm{c}=\frac{1}{2} \rho \mathrm{F} \int_{0}^{\pi}[\mathrm{p}(\xi)]^{2} \mathrm{~d} \xi \\
& \mathrm{d}_{1}=\frac{2 \mathrm{P}}{1} \operatorname{Sin} \frac{\mathrm{k} \pi \mathrm{vt}}{1}
\end{aligned}
$$

For Integral 15, Euler formula gives ${ }^{[2]}$ :

$$
\begin{aligned}
& \frac{\partial}{\partial \tau}\left(\frac{\partial \mathrm{L}}{\frac{\partial \mathrm{q}}{\partial \tau}}\right)-\frac{\partial \mathrm{L}}{\partial \mathrm{q}}=0 \\
& 2 c \omega^{2} \mathrm{q}^{\prime \prime}+2 a \mathrm{a}\left(1+2 \frac{\mathrm{b}}{\mathrm{a}} \mathrm{q}^{2}\right)=\frac{\pi \mathrm{P}}{1} \sin \frac{\mathrm{K} \pi \mathrm{Vt}}{1}
\end{aligned}
$$

Or

$$
\omega^{2} q^{\prime \prime}+\frac{a}{c} q\left(1+2 \frac{b}{a} q^{2}\right)=d \sin \eta \tau
$$

Where: $d=\frac{\pi \mathrm{P}}{2 \mathrm{cl}}, \quad \eta=\frac{\theta}{\omega}, \theta=\frac{\mathrm{k} \pi \mathrm{V}}{1} \quad \omega=\omega_{0}=\sqrt{\frac{\mathrm{a}}{\mathrm{c}}}$ have:

By substitution of $X=\frac{\omega^{2}}{d} q$ in to equation we

$$
\mathrm{X}^{\prime \prime}+\mathrm{X}\left(1+\mathrm{eX}^{2}\right)=\operatorname{Sin} \eta \tau
$$

Where

$$
e=2 \frac{b}{a} \frac{d^{2}}{\omega_{0}^{4}}=\frac{b}{a} \frac{\pi^{2} p^{2}}{21^{2}}
$$

To solve the Duffing Eq. 20, it is assumed:

$$
\begin{aligned}
X & =\sum_{n=1,2,3, \ldots}^{\infty} X_{n} \operatorname{Sin}_{n \eta \tau} \\
& =X_{1} \sin \eta \tau+X_{3} \sin 3 \eta \tau+X_{5} \sin 5 \eta \tau+\ldots
\end{aligned}
$$

By substituting expression 22 at 20 and comparing the coefficients of $\operatorname{Sin}_{n \eta \tau}$. We will get a lot of cubic nonlinear algebraic equations. There is no exact solution for these equations, there for, we emply an approximated method to solve this equation. For this purpose we take the three constraints Eq. 22 and in this case, we will have system of nonlinear equations. Solving that equation by method of Zeidel, we will have:

$$
\left\{\begin{array}{l}
{\left[1-\eta^{2}\right] x_{1}+\frac{3}{4} e x_{1}^{3}=1} \\
X_{3}=\frac{e X_{1}^{3}}{4\left[9 \eta^{2}-1\right]} \\
x_{5}=\frac{3 e x_{1} x_{3}\left[x_{1}+x_{3}\right]}{4\left[25 \eta^{2}-1\right]}
\end{array}\right.
$$

Deformation of beam is given by:

$$
\mathrm{W}(\mathrm{z}, \mathrm{t})=\frac{\mathrm{d}}{\omega_{0}^{2}} \mathrm{p}(\mathrm{z}) \underset{\mathrm{n}=1,3,5}{\sum} \mathrm{X}_{\mathrm{n}} \sin \frac{\mathrm{n} \pi \mathrm{Vt}}{1}
$$

Where:

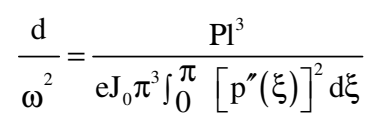

Bending stress at any section of beam is determined by:

$$
\sigma_{\mathrm{Z}}=\mathrm{E}\left(\varepsilon_{\mathrm{Z}}-\frac{2}{27} \mathrm{l}_{2} \frac{\mathrm{E}^{3}}{\mathrm{G}^{3}} \varepsilon_{\mathrm{Z}}^{3}\right)
$$

Bending moment is calculated by:

$$
\begin{aligned}
& M=\iint \sigma_{z} \cdot x d x d y= \\
& E \iint\left[x^{2} \frac{\partial^{2} w}{\partial z^{2}}-\frac{2}{27} 12 \frac{E^{3}}{G^{3}}\left(x \frac{\partial^{2} w}{\partial z^{2}}\right)^{3}\right]= \\
& E J_{\circ} \frac{\partial^{2} w}{\partial z^{2}}\left[1-\frac{2}{27} l_{2} \frac{E^{3}}{G^{3}}\left(x \frac{\partial^{2} w}{\partial z^{2}}\right)^{2}\right]
\end{aligned}
$$


When the load is out of the beam: differential equation of free vibration will be:

$$
\omega^{2} q^{\prime \prime}+\frac{a}{c}\left(1+2 \frac{b}{a} q^{2}\right)=0
$$

Finally, by solving Eq. 27 we find the period of vibration:

$$
\mathrm{T}=4 \cdot \frac{\omega}{\sqrt{\frac{\mathrm{a}}{\mathrm{c}}\left(1+\frac{\mathrm{b}}{\mathrm{a}} \mathrm{Q}^{2}\right)}} \cdot \mathrm{K}(\bar{\theta})
$$

Where, Q-is amplitude of vibration

$$
K(\bar{\theta})=\frac{\pi}{2}\left[1+\frac{1}{4} \sin ^{2} \bar{\theta}+\frac{9}{64} \sin ^{4} \bar{\theta} .+\frac{25}{256} \sin ^{6} \bar{\theta}+. .\right]
$$

And circular acceleration of vibration will be:

$\omega=\frac{\pi}{2} \sqrt{\frac{\mathrm{a}}{\mathrm{c}}\left(1+\frac{\mathrm{a}}{\mathrm{b}} \mathrm{Q}^{2}\right)} \cdot \frac{1}{\mathrm{k}(\bar{\theta})}$

\section{RESULTS AND DISCUSSION}

Now the obtained analytic solution is applied to the following example. Figure 2 shows the cross section of a railway beam. The material of beam is considered steel which is made of a nonlinear material. The specification of the material are as follows:

$\mathrm{I}_{0}=0.3252 \times 10^{-4} \quad \mathrm{~m}^{4} \quad \mathrm{I}_{1}=17.827 \times 10^{-8} \quad \mathrm{~m}^{6}$

$\mathrm{E}=2.1 \times 10^{8} \mathrm{KN} / \mathrm{m}^{2} \quad \mathrm{G}=0.87 \times 10^{8} \quad \mathrm{KN} \mathrm{m}^{-2}$

$1_{2}=0.085 \times 10^{6} \quad \rho=78 \mathrm{KN} \mathrm{m}^{-3}$

$\mathrm{P}=62.5 \mathrm{KN} \quad 1=3.00 \mathrm{~m}$

$\mathrm{k}_{1}=0,500,1000,1500,2000 \quad \mathrm{KN} \mathrm{m}^{-2} \quad \mathrm{k}_{2}=0.00, \mathrm{k}_{2}=0.1$

Then vibration amplitudes are determined for $\mathrm{k}_{1}=1000$ and $\mathrm{k}_{2}=0.1$ are shown in the Table 1. Based on Table 1 resonance curve is drawn (Fig. 3).

When, $\eta^{2}=1$, for $\mathrm{K}_{1}=0.00,500,1000,1500,2000$ and $\mathrm{k}_{2}=0.1$, The deformation, bending stress and bending moment at the middle span is determined and is showed at Table 2.

When $\mathrm{k}_{2}=0.0$ that is nonlinear beam on elastic foundation (Winkler Theorem), in this case, the deformation, stress and bending moment at the middle span is determined and is showed at the Table 3.

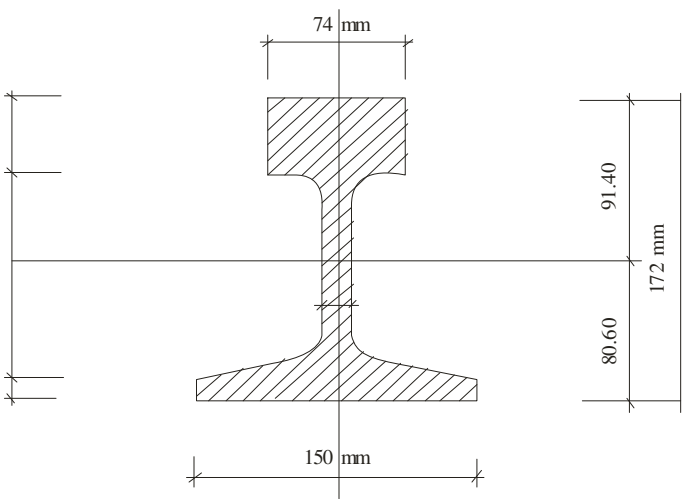

Fig. 2: Section of beam

Table 1: Dynamic coefficients calculated for $\mathrm{k}_{1}=1000, \mathrm{k}_{2}=0.1$ $\mathrm{X}_{1}$

\begin{tabular}{lrrrcl}
$\eta^{2}$ & $\mathrm{X}_{1}^{\mathrm{I}}$ & $\mathrm{X}_{1}^{\mathrm{N}}$ & $\mathrm{X}_{1}^{\mathrm{II}}$ & \multicolumn{1}{c}{$\mathrm{X}_{3}$} & $\mathrm{X}_{5}$ \\
\hline 0.00 & -13.69 & 12.68 & 1.00 & $7.63 * 10^{-3}$ & $1.76^{*} 10^{-4}$ \\
0.6619 & -8.87 & 4.45 & - & -0.1356 & $4.073^{*} 10^{-3}$ \\
1 & -5.59 & & & 0.1665 & $4.81^{-3} 10^{-3}$ \\
2 & -1.0 & & & $4.48 * 10^{-4}$ & $-2.09 * 10^{-7}$ \\
\hline
\end{tabular}

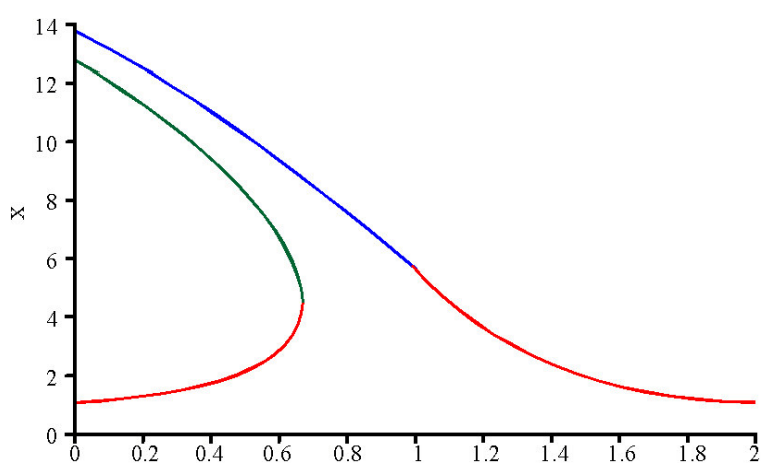

Fig. 3: Resonance curve

Table 2: Deformation, stress and bending moment for $\mathrm{k}_{1}, \mathrm{k}_{2}$

\begin{tabular}{llllll}
\hline $\mathrm{K}_{1}\left(\mathrm{KN} / \mathrm{m}^{2}\right)$ & $\mathrm{e}$ & $\mathrm{X}_{1}$ & $\mathrm{~W}(\mathrm{~cm})$ & $\sigma_{\mathrm{t}}(\mathrm{MPa})$ & $\mathrm{M}(\mathrm{KNm})$ \\
\hline & & - & & & \\
0 & -0.00281 & 707997 & 1.98 & 271.424 & 114.3 \\
500 & -0.00482 & -6.5157 & 1.62 & 263.094 & 103.7 \\
1000 & -0.00463 & -5.5908 & 1.39 & 244.724 & 93.40 \\
1500 & -0.01162 & -4.8593 & 1.23 & 223.969 & 83.80 \\
2000 & -0.01438 & -4.2491 & 1.07 & 203.004 & 75.00 \\
\hline
\end{tabular}

Table 3: Deformation, stress and bending moment for $\mathrm{k}_{1}$

\begin{tabular}{llllll}
\hline $\mathrm{K}_{1}\left(\mathrm{KN} / \mathrm{m}^{2}\right)$ & $\mathrm{e}$ & $\mathrm{X}_{1}$ & $\mathrm{~W}(\mathrm{~cm})$ & $\sigma_{\mathrm{t}}(\mathrm{MPa})$ & $\mathrm{M}(\mathrm{KNm})$ \\
\hline 0.00 & -0.00281 & -7.7997 & 1.98 & 271.424 & 114.3 \\
500 & -0.0034 & -7.3196 & 1.85 & 240.830 & 110.8 \\
1000 & -0.0041 & -6.8468 & 1.73 & 267.789 & 107.2 \\
1500 & -0.00516 & -6.3694 & 1.61 & 261.120 & 102.2 \\
2000 & -0.0062 & -5.8976 & 1.49 & 252.215 & 97.1 \\
\hline
\end{tabular}




\section{CONCLUSION}

It is concluded that when the behavior of material is assumed to be physically nonlinear then:

- The circular frequency of system decreases

- It is shown when The material is physical nonlinear, for $\eta^{2}=\frac{\theta^{2}}{\omega^{2}}=1$, there is no resonance and there is no critical velocity and there are finite values for the deflection, stress and bending moment of the beam

- Values for deformation, stress and bending moments have been obtained for the various $\mathrm{k}_{1}$ and $\mathrm{k}_{2}=0.1$ nonlinear elastic foundation and $\mathrm{k}_{2}=0.0$ (Winkler elastic foundation), The obtained results at $\mathrm{k}_{2}=0.1$ is smaller than that $\mathrm{k}_{2}=0.0$

- Values of $x_{1}$ (dynamic coefficients) decrease due to increase in $\mathrm{k}_{1}$

- For any velocity $\mathrm{V}$ deformation, stress and bending moment can also be analyzed

\section{REFERENCES}

1. Kauderer, H., 1961. Nonlinear Mechanic. Moscow, pp: 777.

2. Babakov, I.M., 1968. Theory of Vibration. Moscow, pp: 560.

3. Glushkov, G., 1974. Engineering Method for Analyzing Strength and Rigidity. Moscow, pp: 356.

4. Kesiliev, V.A., 1972. Structural Mechanic. Moscow, pp: 550. 